\title{
Intrainsular connectivity and somatosensory responsiveness in young children with ASD
}

Michelle D. Failla', Brittany R. Peters², Haleh Karbasforoushan ${ }^{3}$, Jennifer H. Foss-Feig ${ }^{4,5}$, Kimberly B. Schauder ${ }^{6}$, Brynna H. Heflin ${ }^{1}$ and Carissa J. Cascio ${ }^{1,7^{*}}$

\begin{abstract}
Background: The human somatosensory system comprises dissociable paths for discriminative and affective touch, reflected in separate peripheral afferent populations and distinct cortical targets. Differences in behavioral and neural responses to affective touch may have an important developmental role in early social experiences, which are relevant for autism spectrum disorder (ASD).

Methods: Using probabilistic tractography, we compared the structural integrity of white matter pathways for discriminative and affective touch in young children with ASD and their typically developing (TD) peers. We examined two tracts: (1) a tract linking the thalamus with the primary somatosensory cortex, which carries discriminative tactile information, and (2) a tract linking the posterior insula-the cortical projection target of unmyelinated tactile afferents mediating affective touch — with the anterior insula, which integrates sensory and visceral inputs to interpret emotional salience of sensory stimuli. We investigated associations between tract integrity and performance on a standardized observational assessment measuring tactile discrimination and affective responses to touch.
\end{abstract}

Results: Both the thalamocortical and intrainsular tracts showed reduced integrity (higher mean diffusivity) in the ASD group compared to those in the TD group. Consistent with the previous findings, the ASD group exhibited impaired tactile discriminative ability, more tactile defensiveness, and more sensory seeking (e.g., enthusiastic play or repetitive engagement with a specific tactile stimulus). There was a significant relation between intrainsular tract integrity and tactile seeking. The direction of this relation differed between groups: higher intrainsular mean diffusivity (MD) (reflecting decreased tract integrity) was associated with increased tactile seeking in the TD group but with decreased tactile seeking in the ASD group. In the TD group, decreased tactile defensiveness was also associated with higher intrainsular MD, but there was no relation in the ASD group. Discriminative touch was not significantly associated with integrity of either tract in either group.

Conclusions: These results support previous findings suggesting a central role for the insula in affective response to touch. While both discriminative and affective touch and both somatosensory tracts are affected in ASD, the restriction of brain-behavior associations to the intrainsular tract and tactile seeking suggests more complex and perhaps higher-order influence on differences in tactile defensiveness and discrimination.

\footnotetext{
* Correspondence: carissa.cascio@vanderbilt.edu

${ }^{1}$ Department of Psychiatry, Vanderbilt University Medical Center, Nashville, TN

37212, USA

${ }^{7}$ Vanderbilt Kennedy Center, Nashville, TN 37203, USA

Full list of author information is available at the end of the article
}

(c) The Author(s). 2017 Open Access This article is distributed under the terms of the Creative Commons Attribution 4.0 International License (http://creativecommons.org/licenses/by/4.0/), which permits unrestricted use, distribution, and reproduction in any medium, provided you give appropriate credit to the original author(s) and the source, provide a link to the Creative Commons license, and indicate if changes were made. The Creative Commons Public Domain Dedication waiver (http://creativecommons.org/publicdomain/zero/1.0/) applies to the data made available in this article, unless otherwise stated. 


\section{Background}

Autism spectrum disorder (ASD) is characterized by developmental impairments in social communication and restricted and repetitive behaviors [1], which often include differences in sensory responsiveness to environmental stimuli1 [2, 3]. Children with ASD may exhibit patterns of altered sensory reactivity such as hyporesponsiveness [4-6], hyper-responsiveness [7, 8], and/or sensory "seeking"-unusual interest in or fascination with specific sensory stimuli [9]. While these patterns manifest with considerable inter-individual variability and are likely to have different underlying genetic [10] and neural $[11,12]$ mechanisms, recent work has begun to describe how these patterns of behavioral reactivity to sensory stimuli co-occur within individuals [13].

Efforts toward untangling the neural basis of sensory reactivity patterns have focused on auditory and visual modalities, possibly because of their primacy to verbal and nonverbal communication skills affected by ASD. Far less work has been done to understand more proximal sensory systems such as touch, proprioception, and interoception. However, these systems are also of critical importance during social interactions, providing important cues about emotion, attachment, compliance, and intimacy [14]. Tactile responsiveness patterns specifically are linked to social deficits in ASD [6] and may have a central role in our earliest experiences of social communication. Specifically, infant-caregiver interactions that lay the foundation for social reward and secure attachment heavily involve the sense of touch $[15,16]$, which is at an advanced stage of development relative to other sensory systems in neonates [17]. It is during this early window in the first year of life that neural differences in ASD begin to emerge [18]. Thus, the developmental primacy of touch for behaviors relevant to ASD warrants further investigation.

While sensory reactivity is typically assessed with clinical observational measures, discriminative touch has also been increasingly measured in ASD using rigorous psychophysical methods that often give insight into neurobiological mechanisms. While there is not a clear consensus for impaired or enhanced discriminatory ability, some themes have emerged from this work (for a review, see Mikkelsen et al. [19]). Impaired performance on vibrotactile static detection and discrimination tasks suggests impaired lateral inhibition in the somatosensory cortex [20,21], as do impairments in amplitude discrimination and the absence of expected effects of a habituating stimulus [20, 22]. These sensory indications of impaired cortical inhibitory mechanisms have been tied directly to reduced GABA in the sensorimotor cortex in ASD using spectroscopy [23] and support the excitatory/ inhibitory imbalance hypothesis of ASD [24, 25]. The well-established autism candidate gene GABRB3 is associated with differences in tactile sensitivity [26, 27], providing further support for a role of GABA dysfunction in the somatosensory differences common in individuals with ASD.

Distinct but overlapping neural pathways support the discriminative and affective aspects of touch. Extensive work suggests that discriminative touch (e.g., touch used to determine the shape or texture of an object) is primarily mediated by the thalamocortical projections to the somatosensory cortex [28-30]. However, a separate system that is believed to mediate affective touch (e.g., touch used to elicit an emotional response or to communicate social affiliation) has recently been described. In this system, small-diameter, unmyelinated peripheral fibers, known as C-tactile (CT) afferents, respond preferentially to slow, stroking touch with light to moderate pressure, suggesting they are "tuned" to social/affective (in contrast to discriminative) touch [31-33]. Functional magnetic resonance imaging (fMRI) studies in patients lacking large-diameter myelinated tactile afferents demonstrate that CT fibers project selectively to posterior insular cortex [32, 34], a cortical target of multimodal sensory input that is associated with changes in affective state, including visceral sensation, temperature, and pain, further distinguishing this affective touch system from discriminative touch.

The insula is part of a complex cortical structure with a heterogeneous functional anatomy along its anteriorposterior axis. While the posterior insula receives somatosensory and visceral input, the anterior insula comprises heavily reciprocal projections with prefrontal and limbic regions $[35,36]$. This functional organization may be the basis of a caudo-rostral hierarchical processing stream by which sensory cues are received and integrated with emotional signals to form progressively higher-order representations of proximal (tactile and interoceptive) sensory information and its affective significance [37]. This model is supported by fMRI evidence of strong connectivity between the anterior and posterior insula [38]. There is growing evidence that the anterior insula, whose role as a hub of the salience network is to engage neural networks in response to emotionally important sensory stimuli [39], is affected in ASD [40, 41]. However, the connectivity between the affective anterior insula and sensory posterior insula has not been examined in ASD, nor has the relation between the insula and sensory-affective sequelae that are increasingly considered foundational to the disorder $[42,43]$.

In this study, we hypothesized that the integrity of structural connectivity in somatosensory and insular regions associated with discriminative and affective touch, respectively, would relate to observed sensory behaviors in a standardized assessment of tactile discrimination and affective responses to touch in young children with 
ASD. Given the functional posterior-anterior gradient of the insula and the clear differences in affective touch perception $[8,44]$ and interoception [45-47] in ASD, we specifically examined the structural integrity of intrainsular white matter as a neural substrate for affective response to touch in young children with ASD. As a comparison, we also examined a thalamocortical tract between the ventroposterolateral (VPL) thalamus and primary somatosensory cortex (SI), which is associated with discriminative, rather than affective, touch processing. With a growing body of literature describing differences in discriminative [19] as well as affective response [44] to touch in ASD, we hypothesized that structural connectivity in multiple tracts might differ in ASD [48, 49]. However, given the functional roles of these regions, we predicted that connectivity between the anterior and posterior insula would specifically relate to aberrant affective response to touch in individuals with ASD, while connectivity between the VPL thalamus and SI would uniquely relate to aberrant touch discrimination in individuals with ASD.

\section{Methods \\ Sample}

Twenty-nine children with ASD and 26 typically developing (TD) children between the ages of 5 and 8 years were recruited into the study. This sample is the same as that reported by Pryweller et al. [12]. After excluding participants with poor image quality resulting from excessive motion or scanner/acquisition errors $(\operatorname{ASD} n=5$; TD $n=1)$, the final sample included 23 children with ASD (6.61 years $\pm 0.89,2$ females) and 24 children with TD (6.58 years $\pm 1.13,4$ females). Participants in the ASD group were recruited from the university medical center and surrounding community, and a diagnosis of ASD was confirmed with research-reliable administration of the Autism Diagnostic Observation Schedule (ADOS) [50], the algorithm items of the Autism Diagnostic Interview-Revised (ADI-R) [51], and the judgment of a licensed clinical psychologist based on DSM (4th ed.; DSM-IV) [52] criteria. Participants in the TD group were excluded if they had a diagnosed psychiatric or learning disorder or a first-degree relative with ASD. Additionally, TD participants were screened using the Social Communication Questionnaire (SCQ) [53] and the Child Behavior Checklist (CBCL) [54] to rule out risk for ASD and other psychiatric conditions. All participants were screened and excluded for any genetic and neurological problems, previous head injuries, and MRI contraindications.

Participants' cognitive ability was assessed by trained research assistants using the Kaufman Brief Intelligence Test, Second Edition [55] $(n=47$; KBIT-2) or WISC-IV [56] $(n=1$; Weschsler et al. 2003), or estimated $(n=1)$ with the Mullen Scales of Early Learning (MSEL) [57], dependent on the language level of the participant. Although the groups did not differ on sex $\left(\chi^{2}(1)=.145\right.$, $p=.70)$ or chronological age $(t(43.3)=.085, p=.932)$, Full Scale IQ estimate was significantly higher in the TD group $(t(28.8)=-4.01, p=.0004)$. See Table 1 for a summary of participant characteristics. Because IQ differed between groups, it was considered as a potential covariate; however, IQ was not significantly correlated with any diffusion variables.

\section{Sensory assessment}

Each child was administered the Tactile Defensiveness and Discrimination Test-Revised [12] (Baranek, 2010, unpublished manual). The Tactile Defensiveness and Discrimination Test-Revised (TDDT-R) is a structured observational assessment including internally controlled (active) touch (e.g., using the palm to rub chalk drawings from a square of carpet, digging pennies out of a box of sand) and externally controlled (experimenter-administered, passive) touch (e.g., light touch to the arm, face, or hand with a cotton swab, air puffs administered to the nape of the neck). Affective behavioral responses are scored for (1) tactile defensiveness both to passive, externally controlled/experimenter-administered touch and to active, internally controlled touch (i.e., haptic exploration of materials such as fabric, sand, or putty) and (2) sensory seeking responses (e.g., squealing with delight while enthusiastically playing with sand, repetitively touching fabric), reflecting unusual interest in tactile stimuli. Defensiveness to externally controlled items is scored using a Likert scale from $0-3$, with 0 representing no aversive reaction, and 3 representing negative affect that includes crying or retreating from the stimulus. Defensiveness to internally controlled items is a composite of two scores, one assessing approach or avoidance of the stimulus (0-2 scale) and the other a binary score assessing aversive reaction. Total defensiveness is calculated as a composite (sum) variable collapsing across defensiveness scores for both internally and externally

Table 1 Sample demographics

\begin{tabular}{llll}
\hline & ASD & TD & $p$ value \\
\hline N scanned & 29 & 26 & - \\
N retained after QA & 23 & 24 & - \\
Mean age in years (SD) & $6.61(.89)$ & $6.58(1.13)$ & 0.932 \\
FSIQ & $98(19.3)$ & $117(12.1)$ & .0004 \\
Mean QA rating (SD) & $4.21(0.60)$ & $4.54(0.64)$ & 0.08 \\
N female & 2 & 4 & 0.703 \\
Mean ADOS severity score (SD) & $7.65(2.06)$ & - & - \\
\hline
\end{tabular}

$p$ values represent independent sample $t$ tests for age, QA, and FSIQ, chi-square test for sex. Significant $p$ values are in italics

$S D$ standard deviation, FSIQ Full Scale IQ, ADOS Autism Diagnostic Observation Schedule, ASD autism spectrum disorder, TD typically developing 
controlled stimuli. For internally controlled items, sensory seeking is scored using a binary present/absent score. Discriminative behavioral responses are scored for passive, externally controlled touch (i.e., localization of the body part touched by experimenter) and active, internally controlled touch (i.e., correct haptic identification of shapes or forms handled by the participant).

\section{Image acquisition and preprocessing}

All images were acquired during a single scan session on a 3 Tesla Philips Achieva MRI scanner (Philips Healthcare, Inc., Best, Netherlands). During scanning procedures, participants wore foam earplugs in both ears and Philips headphones to attenuate noise and watched a video of their choice for the duration of the scan. A highresolution T1-weighted anatomical volume $(\mathrm{TR}=9 \mathrm{~ms}$, $\mathrm{TE}=4.6 \mathrm{~ms}, \mathrm{FOV}=256 \mathrm{~mm}[2], 1 \mathrm{~mm}$ isotropic voxels, 170 sagittal slices, 6 min $30 \mathrm{~s}$ duration) was collected to provide a template for image registration. Diffusionweighted data were acquired using a high angular resolution diffusion imaging (HARDI) sequence $(2.5 \mathrm{~mm}$ [2] isotropic voxels, 50 axial slices, $14 \mathrm{~min} 34 \mathrm{~s}$ duration). Ninety-two diffusion directions $(b=1600 \mathrm{~s} / \mathrm{mm}[2])$ and one T2-weighted volume ( $b=0 \mathrm{~s} / \mathrm{mm}[2])$ were collected.

Images were visually inspected for common artifacts such as fat shift and ghosting and underwent standard preprocessing and quality assurance procedures that incorporated head motion, artifact propensity, variance, and bias of estimated measures [58]. A QA rating that weighted each of these measures was assigned, with values between 1 and 5 . Only scans with ratings equal to or higher than 3 were included in the analysis (as in (12)). The final sample included in the analysis did not significantly differ in this comprehensive QA metric by group $(t(45)=-1.79, p=.08)$. HARDI data were eddy current-corrected, motion-corrected, and skull-stripped using FMRIB Software Library (FSL) [59, 60]. Fractional anisotropy (FA) maps, eigenvectors, and eigenvalues were created through the DTIFIT module within FSL.

\section{Probabilistic tractography}

Diffusion parameters were estimated at each voxel through the Bayesian Estimation of Diffusion Parameters Obtained using Sampling Techniques (BEDPOSTX) tool in FSL, which uses Markov chain Monte Carlo sampling techniques to estimate diffusion parameters [61]. Within the right hemisphere, two tracts were defined: an intrainsular tract between anterior and posterior insula and a thalamocortical tract between the VPL nucleus of the thalamus and SI (Fig. 1). For the intrainsular tract, anterior and posterior insular seeds (Fig. 1) were traced with a protocol similar to Farb et al. [38] on a pediatric template derived from 324 children ages 4.5-8.5 [62, 63]. The insula was divided into distinct regions based on

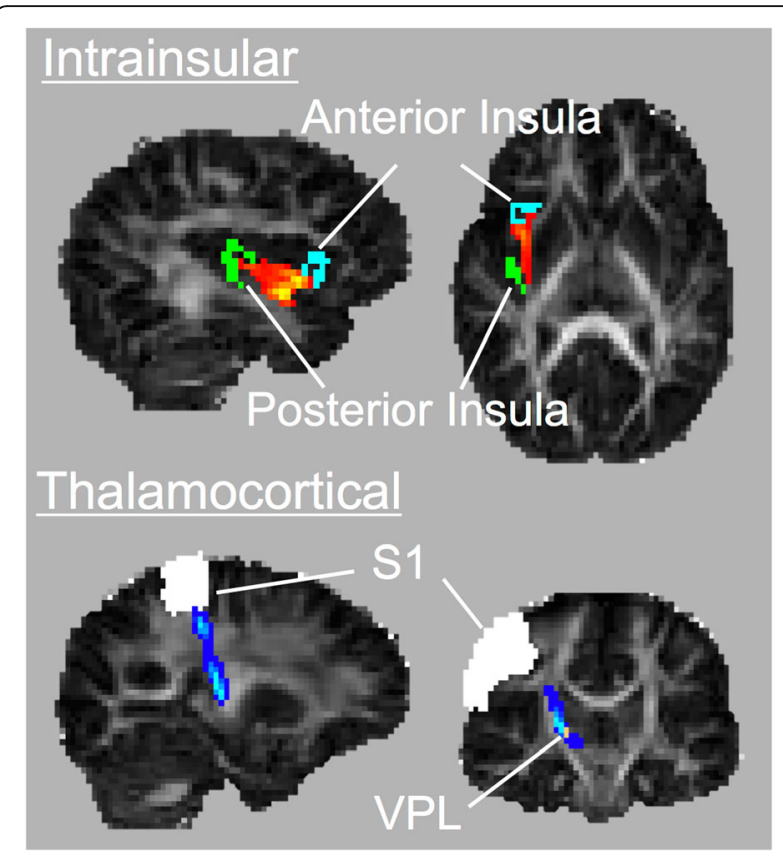

Fig. 1 Representative probabilistic distributions of each tract and regions of interest (ROI) used as seed and termination regions for each tract, rendered on fractional anisotropy maps. Intrainsular tract (top, red-orange) was seeded from the posterior insula (green) to the anterior insula (b/ue). The thalamocortical tract (bottom, blue-light blue) was seeded from the ventral posterolateral nucleus of the thalamus (VPL, yellow) to primary somatosensory cortex (SI, white)

cytoarchitectonically defined subdivisions among the insular gyri. The anterior seed mapped onto the anterior accessory gyrus, and the posterior seed mapped onto the posterior long gyrus of the insula. For the thalamocortical tract, VPL was defined by the Oxford Thalamic Connectivity Atlas [64], and SI was defined as the post-central gyrus from the Laboratory of Neuroimaging (LONI) probabilistic atlas gray matter tissue map, excluding voxels below a threshold of $15 \%$ probability [65]. VPL and SI ROIs were then registered to the pediatric template, and all ROIs subsequently were registered into native space for each subject using a combination of FSL's linear image registration tool and linear and non-linear Advanced Normalization Tools (ANTs) [66, 67]. Using these seeds and diffusion parameters generated by BEDPOSTX, probabilistic tracking was conducted using FSL's ProbTrackX (curvature threshold $=0.2 ; 5000$ samples) one-way condition with VPL and posterior insula as seeds and SI and anterior insula as respective waypoints and termination masks. For the larger thalamocortical tract, lowprobability voxels were rejected at a threshold of $1 \%$, similar to previous studies $[68,69]$. Tracts were visually inspected to confirm that these thresholds reduced noise while producing viable tracts for analysis. Mean fractional anisotropy (FA), mean diffusivity (MD), and volume of each resultant tract were calculated for each thresholded 
tract. Tract volumes were normalized for each participant by dividing the value by the total brain volume (total white matter, gray matter, and cerebrospinal fluid on skullstripped T1-weighted anatomical images).

\section{Statistical analysis}

Our primary questions were (1) whether the integrity of white matter in the intrainsular and thalamocortical tracts differs between children with ASD and TD and (2) whether white matter integrity differences in one or both tracts are associated with aberrant affective and discriminative behavioral responses to touch in individuals with ASD, as measured by the TDDT-R. For all diffusion tensor imaging (DTI) variables (FA, MD, and volume in each of the two tracts), we removed outliers (0-2 datapoints per variable) based on values that were extreme (3 times interquartile range). To address question 1, we assessed group differences in DTI variables (FA, MD, volume) for each tract using two sample $t$ tests. Although it was not a primary research question, we also assessed group differences in variables derived from the TDDT-R: tactile haptic form perception (discriminative touch), tactile defensiveness (negative affective response to touch) to both internally and externally controlled touch, and tactile seeking (presumed positive affective response to touch). Mann-Whitney tests were used for these comparisons because TDDT-R variables were non-normally distributed (Table 2).

To address question 2, we performed nonparametric (Spearman) correlations only between variables (both DTI and behavioral) that had significant differences by group (MD in both tracts and all three TDDT-R variables, see results below). Limiting comparisons to those for which the groups differed significantly allowed us to address the contingency of question 2 on question 1 and to reduce the number of tests performed. To understand how the group interacted with MD in each tract to influence behavior, we conducted a multiple linear regression with the relevant behavioral response score from the TDDT-R as the dependent variable.

To address the possibility of type I error due to multiple comparisons, we conducted permutation testing for significant group comparisons of DTI and TDDT variables as well as TDDT and DTI correlations [70]. A null distribution was calculated for each test statistic across each permutation $(n=5000)$. A $p$ value was determined based on the proportion of more extreme test statistics compared to the observed test statistic $(\alpha=$ $0.05)$. The second $p$ value reported for the statistically significant findings reported below reflect this corrected value.

\section{Results \\ Tractography}

For both the intrainsular and thalamocortical tracts, MD was significantly higher in the ASD group (intrainsular MD $t=2.14, p=.039$ (permuted $t$ distribution $(n=5000)$, $p=0.031)$ ); thalamocortical MD $t=2.3, p=.026$ (permuted $t$ distribution $(n=5000), p=0.024)$; Fig. $2 \mathrm{~b}, \mathrm{e})$. Intrainsular volume was marginally lower in the ASD group ( $t=-1.77, p=.084)$, and both intrainsular $(t=$ -1.61, $p=.116)$ and thalamocortical $(t=-1.35, p=.183)$ FA showed slight trends to be lower in the ASD group (Fig. 2), consistent with a pattern of reduced tract integrity in ASD. There were no significant group differences or trends in thalamocortical volume.

\section{Discriminative and affective responses to touch}

As measured by the TDDT-R, the ASD group exhibited significantly poorer internally controlled (form) haptic discrimination $(W=384.5, p=.0215$ (permuted $W$ distribution $(n=5000), p=0.017))$ relative to the TD group. In ASD relative to $\mathrm{TD}$, defensiveness to internally controlled tactile stimuli (haptic exploration of materials such as sand or putty) was significantly higher ( $W=375, p=.0339$, (permuted $W$ distribution $(n=5000)$, $p=0.028)$ ), and defensiveness to externally controlled touch (e.g., experimenter touch with a cotton swab) was also higher $(W=378.5, p=.0257$ (permuted $W$ distribution $(n=5000), p=0.026))$. The ASD group exhibited significantly greater tactile seeking behaviors $(W=438.5, p=.0003$ (permuted $W$ distribution ( $n=$ 5000), $p<0.001)$ ) relative to the TD group.

Table 2 Median (interquartile range) scores by group on the Tactile Defensiveness and Discrimination Test-Revised (TDDT-R)

\begin{tabular}{lllll}
\hline Tactile ability/response & TDDT-R & ASD & TD & $p$ value \\
\hline Discrimination & Form perception & $12.17(3.92)$ & $9.71(4.22)$ & 0.0125 \\
Affective response & Defensiveness to externally (experimenter) controlled touch & $0.1875(.03)$ & $0.0625(.13)$ & 0.0257 \\
& Defensiveness to touching objects or materials & $0.2143(.25)$ & $0.1429(.14)$ & 0.0339 \\
& Tactile seeking & $0.2857(.14)$ & $0.0000(.14)$ & .0003 \\
\hline
\end{tabular}

Externally controlled touch is the touch administered by the examiner; internally controlled touch is tactile exploration of objects or materials by the participant. For form perception, higher scores indicate poorer performance (i.e., more time manipulating objects needed to accurately discriminate form). For all other tests, higher scores indicate more observable affective responses to sensory probes. $p$ values represent Mann-Whitney $U$ tests. Significant $p$ values are in italics ASD autism spectrum disorder, TD typically developing 


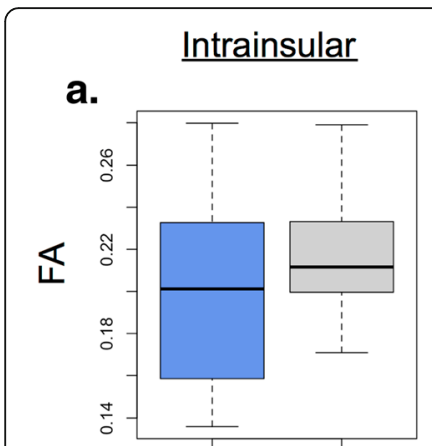

\section{Thalamocortical}

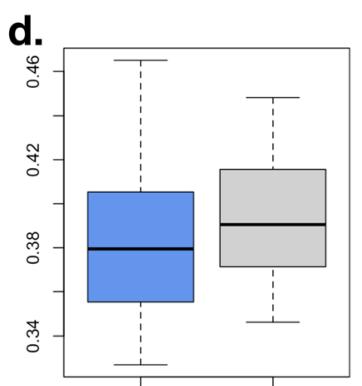

b.

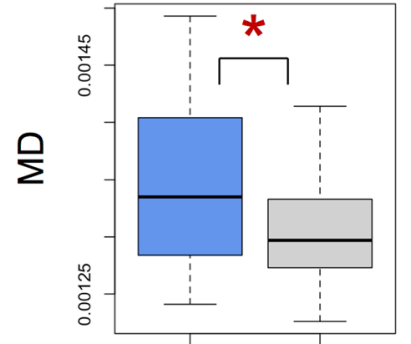

c.

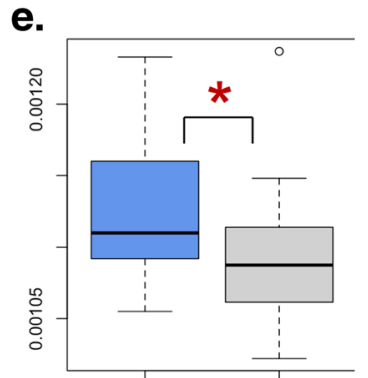

f.

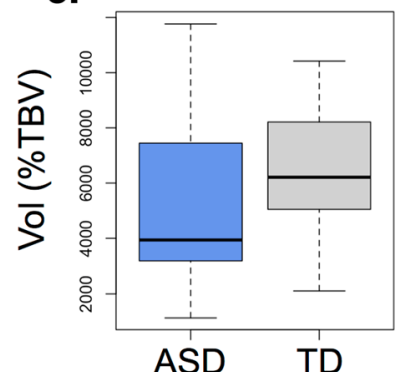

Fig. 2 Group differences in tractography. a-c Boxplots by group of intrainsular tract variables. a While FA was lower in individuals with ASD, this was not significantly different compared to that in TD $(p=.116)$. $\mathbf{b}$ MD was significantly higher in the ASD group, compared to that in the TD group $\left({ }^{*} p=.039\right)$. $\mathbf{c}$ Volume was lower in individuals with $A S D$, but was not significantly different compared to that in TD $(p=.084)$. $\mathbf{d}-\mathbf{f}$ Boxplot distributions by group of thalamocortical tract variables. $\mathbf{d}$ While FA was lower in individuals with ASD, this was not significantly different compared to that in TD ( $p=.183$ ). e MD was significantly higher in the ASD group, compared to that in the TD group $\left({ }^{*} p=.026\right)$. $\mathbf{f}$ There was no significant group difference in volume. ASD autism spectrum disorder, TD typical development, FA fractional anisotropy, MD mean diffusivity, TBV total brain volume

\section{Association between tactile behavioral responses and mean diffusivity}

A significant positive association was observed between intrainsular MD and tactile seeking behavior in the TD group $(\rho(22)=0.49, p=0.0387$ (permuted $\mathrm{S}$ distribution $(n=5000), p=0.016))$, whereas this association was negative in the ASD group $(\rho(20)=-0.50, p=0.0185$ (permuted S distribution ( $n=5000), p=0.016$, Fig. 3$)$ ). A Fisher $r$-to- $z$ transformation confirmed that these correlation coefficients were significantly different $(z=$ $3.47, p=.0005)$. Given the opposite directions of these

associations, we then tested for a group*intrainsular MD interaction using a multiple linear regression with tactile seeking as the dependent variable. Full Scale IQ was also included in the model given the group differences in IQ (see Table 1). The model verified significant main effects of group on seeking and showed a main effect of insula MD $\left(t(36)=-3.676, p=.0008\right.$, adjusted total model $r^{2}=$ 0.4647), but not thalamocortical MD or Full Scale IQ (FSIQ), on tactile seeking (see Table 3). There was a significant interaction between group and insula $\mathrm{MD}$ $\left(t(36)=3.470, p=.0014\right.$, adjusted total model $r^{2}=$ 0.4647).

Neither group exhibited significant associations between tactile discrimination scores and MD in either tract. The TD group showed a negative association between tactile defensiveness and MD in the intrainsular tract $(\rho(22)=0.42, p=0.0402$ (permuted S distribution $(n=5000), p=0.042)$ ); this association was absent in the ASD group.

\section{Discussion}

The goal of this study was to assess the integrity of structural connectivity in somatosensory regions associated with affective and discriminative touch [31] in young children with ASD and to relate it to observed sensory behaviors in a standardized assessment of discriminative and affective responses to touch (Baranek, 2010, unpublished manual). Using the TDDT-R, we observed widespread differences in responses to both affective and discriminative touch in young children with ASD, as has been reported previously $[6,8,10,11,20$ $22,71-73]$. In the ASD group, we found reduced integrity (increased MD) of the white matter tract connecting the posterior and anterior insula. Based on the roles of these two regions in somatic sensation [32] and affective evaluation [39], respectively, we hypothesize that this tract is likely important for the emotional evaluation of somatic sensory input. The posterior insula receives input from CT afferents [32], a peripheral system that mediates affective touch by responding preferentially to slow, gentle stroking touch on hairy skin. CT afferents are absent from the palmar surface of the hand, suggesting they do not have a role in discriminative touch [74]. Emerging research suggests that individuals with ASD or heightened autistic traits show diminished response to affective touch all along the anterior-posterior axis of the insula [75-77]; the reduced integrity of the intrainsular tract shown by the current study may be a structural basis for this diminished response.

We noted disparate relationships in the two groups between sensory seeking and the integrity of the intrainsular tract, such that higher levels of tactile seeking were associated with more intact (lower MD) intrainsular white matter in the ASD group and with less intact 


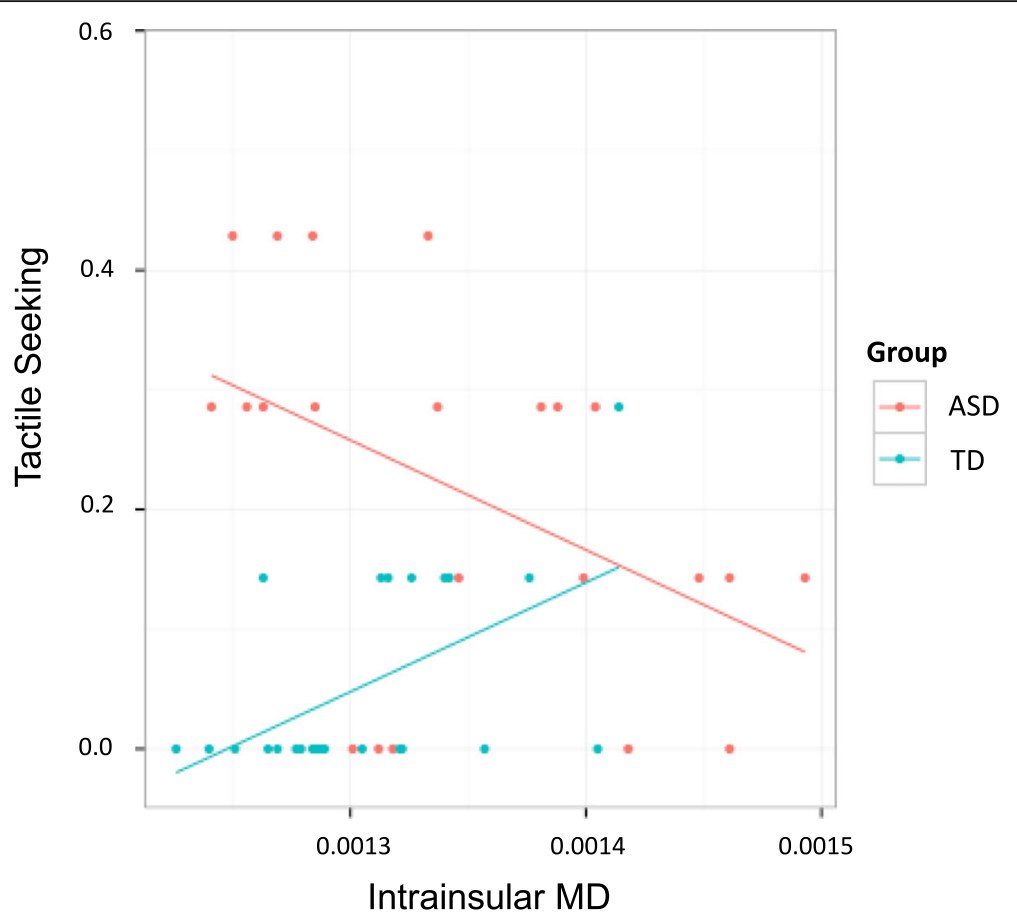

Fig. 3 Associations between tactile seeking as measured by the Tactile Defensiveness and Discrimination Test-Revised (TDDT) and intrainsular tractography. Regression lines for ASD (red) and TD (blue) groups are shown. Significant (Spearman) correlations between intrainsular MD and TDDT-R sensory seeking were observed in the ASD group $(\rho(20)=-0.50, p=0.0185)$ and the TD group $(\rho(22)=0.49, p=0.0387)$

(higher MD) in the TD group. The frequency of sensory seeking is low in the TD group, and thus, the association with compromised white matter in the insula may reflect aberrant affective responses to touch in typical children and is likely to be of an entirely different etiology than sensory seeking in ASD. The link between sensory seeking and better white matter integrity in the ASD group is intriguing given the debate about the valence of affect in sensory seeking behavior in ASD [9]. While the repetitive nature of the behavior suggests that it is intrinsically reinforcing and there is empirical [78] and autobiographical [79] evidence for association with positive affect, there is also evidence linking the behavior with negative [80] or neutral [9] affect. The association of tactile seeking with greater integrity of a neural pathway that supports positive affective touch supports, but does not prove, the view that sensory seeking reflects positive affect.

It has been hypothesized that sensory seeking behaviors may reflect a compensatory strategy for reduced sensory input [81] or, alternatively, may serve as a coping strategy in response to overwhelming sensory experiences [82]. The possibility that tactile seeking is a compensatory strategy for diminished sensory input may be consistent with association between increased seeking and reduced integrity of the intrainsular tract in the TD group. However, in the ASD group, increased seeking was associated with greater integrity of the intrainsular tract. This unexpected profile in ASD-of greater sensory seeking behaviors relating to better integrity of

Table 3 Linear regression model of tactile seeking (as measured on the Tactile Defensiveness and Discrimination Test-Revised (TDDT-R)) with group, FSIQ, and MD. Total model variance explained, $r^{2}=0.4647$

\begin{tabular}{lcccc}
\hline Variable & Estimate & Standard error & $t$ value & $p$ value \\
\hline Full Scale IQ & -0.0014 & 0.0010 & -1.299 & 0.2023 \\
Group & -3.073 & 0.9852 & -3.119 & 0.0036 \\
Insula MD & -2732.22 & 743.35 & -3.676 & 0.0008 \\
Thalamocortical MD & -416.37 & 1080.47 & -0.385 & 0.7022 \\
Group*Insula MD & 1856.07 & 534.95 & 3.470 & 0.0014 \\
Group*Thalamocortical MD & 438.42 & 685.19 & 0.640 & 0.5263 \\
\hline
\end{tabular}

Significant $p$ values are in italics 
the intrainsular tract-suggests that a more typical level of throughput of sensory information to the salience network may increase the salience of the input disproportionately in ASD, leading to repetitive engagement with a sensory stimulus. Thus, for those individuals with ASD for whom the intrainsular tract is relatively more intact, seeking behaviors could be more effective at increasing sensory input, and thus more selfreinforcing-manifesting as increased intensity or frequency of seeking in a positive feedback loop. This is consistent with the ideas that sensory seeking may overlap with repetitive behaviors more broadly $[6,83]$ and that many repetitive behaviors appear to be reinforced by affective and reward circuitry [84, 85]. The relatively binary distribution of seeking behavior and its association with a neurobiological variable in our sample suggests its potential for distinguishing meaningful subtypes based on sensory reactivity. Assessing such a subtype in a larger sample might provide additional information about the adaptive function of sensory seeking.

We also observed an association within the TD group between intrainsular tract integrity and overall tactile defensiveness. Somatic input to posterior insula is not limited to the pleasant touch described by Olausson and colleagues; the region responds to interoceptive input [86], unpleasant and painful touch as well [87-89]. Higher defensiveness in the TD group was associated with lower MD, which reflects greater integrity between the posterior and anterior insula. Thus, it is plausible that for TD children, enhanced throughput of unpleasant feelings from the posterior insula to the anterior insula resulted in greater tactile defensiveness. This result should be interpreted cautiously, however, since defensiveness was infrequent in the TD group relative to the ASD group and we did not observe a similar association in the ASD group. The specificity of association between intrainsular connectivity and (presumed) pleasant affective response to touch (seeking) in the ASD group to sensory seeking is of note, suggesting that different neural pathways may contribute to the perception of touch as either pleasant or unpleasant in the altered sensory experience of individuals with autism.

Finally, we did not find any associations between discriminative touch (form perception) and either thalamocortical or intrainsular MD. Indeed, we would not have expected to observe associations between discriminative touch and intrainsular integrity, given that somatosensory projections to the posterior insula are limited to affectively relevant inputs, such as social touch [31] and interoception [37]. While we did predict that group differences in this kind of discriminative touch may be modulated by thalamocortical tract integrity, other neural correlates of form discrimination have also been described [90]. A recent study implicates the serotonergic system in the differentiation of affective versus discriminative touch [91]. Given the importance of serotonin for modulating sensory cortical responses [92, 93], the implication of serotonin in ASD [94, 95], and evidence that the variation in this system specifically impacts somatosensory processing in ASD [10], the relations between autism, serotonin, and affective responses to touch, merit further investigation. Given previous work implicating GABA in altered touch perception $[23,26]$ in ASD and the modulatory role of serotonin on GABA signaling [96], the interaction of these two neurotransmitter systems in the context of somatosensory perceptual differences in ASD also warrants further study.

Our study had several important strengths, including the use of a standardized observational measure of tactile responsiveness that included quantification of both discrimination and affective response, a high angular resolution DTI sequence that is optimal for tractography, a rigorous QA procedure, and a relatively large and young sample of children with ASD. We utilized a narrow age band of 5-8 years, which is well before pubertal changes in white matter, increases the homogeneity of the sample, and is the earliest developmental look at these tracts in school-age children with ASD. Our study had limitations as well, including the inability to extrapolate our results beyond this narrow age band or to individuals with ASD who could not complete an MRI scan. Ceiling effects or limited variability in some TDDT-R scores for the TD group for some variables may have also hampered our ability to find additional brain-behavior correlations. Finally, while the narrow age band was a strength in some respects, it also limits the ability to extrapolate our findings to the broader population of individuals with ASD. Future research should focus heavily on characterizing behavioral and neural responses to affective touch earlier in development, as the primacy of touch in infancy for a foundation of social reward is well established [97], but not well studied in infants at risk for developing ASD. Understanding how the intersection of perception and affect-both of which have fairly well-characterized neural circuitry-gives rise to the more complex behavioral symptoms of ASD will also depend on strong translational ties between basic and clinical neuroscience. Ultimately, the combination of prospective longitudinal studies of at-risk infants and better cross-talk between basic and clinical researchers is expected to have a strong impact on understanding the pathophysiology of ASD and advancing evidence-based treatment approaches [43].

\section{Conclusions}

This study finds diminished white matter integrity in a group of children with ASD in two tracts conveying 
somatosensory information. One tract linked the somatosensory VPL nucleus of the thalamus with SI and primarily carries detailed information for discriminative touch. The other tract linked the posterior insula with the anterior insula and would be expected to convey information about the affective nature of somatosensory input. Consistent with previous reports, both discriminative and affective responses to touch were affected in children with ASD. Tactile seeking-defined as enthusiastic and repetitive engagement with a specific sensory stimulus-was associated with the integrity of the intrainsular tract, and the direction of this association differed by group. The findings reported here contribute to our understanding of the neural basis of emotional responses to touch in autism.

\section{Abbreviations \\ ADI-R: Autism Diagnostic Interview-Revised; ADOS: Autism Diagnostic Observation Schedule; ANTs: Advanced Normalization Tools; ASD: Autism spectrum disorder; BEDPOSTX: Bayesian Estimation of Diffusion Parameters Obtained using Sampling Techniques; CBCL: Child Behavior Checklist; CT: C-tactile; DSM: Diagnostic and Statistical Manual (American Psychiatry Association); DTI: Diffusion tensor imaging; FA: Fractional anisotropy; fMRI: Functional magnetic resonance imaging; FSL: FMRIB Software Library; HARDI: High angular resolution diffusion imaging; KBIT: Kaufman Brief Intelligence Test; MD: Mean diffusivity; MRI: Magnetic resonance imaging; MSEL: Mullen Scales of Early Learning; QA: Quality assurance; ROI: Region of interest; SCQ: Social Communication Questionnaire; SI: Primary somatosensory cortex; TBV: Total brain volume; TD: Typically developing; TDDT-R: Tactile Defensiveness and Discrimination Test-Revised; VPL: Ventroposterolateral nucleus of the thalamus; WISC-IV: Weschsler Intelligence Scales for Children. 4th Edition}

\section{Acknowledgements}

The authors thank Dr. Norman Farb for providing the intrainsular ROI tracing protocol and Dr. Grace Baranek for providing the laboratory for training and guidance in the use of the TDDT-R.

\section{Funding}

This work was supported by NIH grants R01MH102272, K01MH090232, UL1 TR000445, and U54 HD083211/2P30HD015052.

\section{Availability of data and materials}

The datasets used and/or analyzed during the current study are available from the corresponding author on reasonable request.

\section{Authors' contributions}

MDF performed the bulk of the image processing, data analysis, and writing of the manuscript. JHF and KBS administered and scored the TDDT-R, assisted with the acquisition of the scans, and contributed to the writing and editing of the manuscript. BHH assisted with the clinical and cognitive testing as well as scan acquisition. HK developed and implemented a processing pipeline for probabilistic tractography; BRP and MDF refined this pipeline and processed the images. CJC supervised the data collection, analysis, and interpretation and writing of the manuscript. All authors read and approved the final manuscript.

\section{Competing interests}

The authors declare that they have no competing interests.

\section{Consent for publication}

Not applicable.

\section{Ethics approval and consent to participate}

This study was conducted with the approval of the Vanderbilt Human Research Protection Program and Institutional Review Board. Informed consent was obtained from the parents before the children were enrolled in the study. In most cases, the children also gave informed assent.

\section{Publisher's Note}

Springer Nature remains neutral with regard to jurisdictional claims in published maps and institutional affiliations.

\section{Author details}

'Department of Psychiatry, Vanderbilt University Medical Center, Nashville, TN 37212, USA. ${ }^{2}$ South Carolina Department of Mental Health, Columbia, SC 29202, USA. ${ }^{3}$ Interdepartmental Neuroscience (NUIN) PhD Program, Northwestern University, Evanston, IL 60208, USA. ${ }^{4}$ Seaver Autism Center for Research and Treatment, Icahn School of Medicine at Mt. Sinai, New York, NY 10029, USA. ${ }^{5}$ Department of Psychiatry, Icahn School of Medicine at Mt. Sinai, New York, NY 10029, USA. ${ }^{6}$ Department of Psychology, University of Rochester, Rochester, NY 14611, USA. `Vanderbilt Kennedy Center, Nashville, TN 37203, USA.

Received: 17 January 2017 Accepted: 17 May 2017

Published online: 13 June 2017

\section{References}

1. American Psychiatric Association. Diagnostic and Statistical Manual of Mental Disorders (5th ed)_DSM 5. American Psychiatric Publishing; 2013.

2. Baranek GT, David FJ, Poe MD, Watson LR. Discriminating sensory features in young children with autism, developmental delays, and typical development. J Child Psychol Psychiatry. 2006;47:591-601.

3. Dawson G, Watling R. Interventions to facilitate auditory, visual, and motor integration in autism: a review of the evidence. J Autism Dev Disord. 2000; 30:415-21.

4. Baranek GT, Watson LR, Boyd BA, Poe MD, David FJ, McGuire L. Hyporesponsiveness to social and nonsocial sensory stimuli in children with autism, children with developmental delays, and typically developing children. Dev Psychopathol. 2013;25(2):307-20.

5. Ben-Sasson A, Hen L, Fluss R, Cermak SA, Engel-Yeger B, Gal E. A metaanalysis of sensory modulation symptoms in individuals with autism spectrum disorders. J Autism Dev Disord. 2009;39(1):1-11.

6. Foss-Feig JH, Heacock J, Cascio CJ. Tactile responsiveness patterns and their association with core features in autism spectrum disorders. Res Autism Spectr Disord. 2012;6:337-44.

7. Baranek GT, Boyd BA, Poe MD, David FJ, Watson LR. Hyperresponsive sensory patterns in young children with autism, developmental delay, and typical development. Am J Ment Retard. 2007;112(4):233-45.

8. Cascio CJ, Lorenzi J, Baranek GT. Self-reported pleasantness ratings and examiner-coded defensiveness in response to touch in children with ASD: effects of stimulus material and bodily location. J Autism Dev Disord. 2016; 46(5):1528-37.

9. Kirby AV, Little LM, Schultz B, Baranek GT. Observational characterization of sensory interests, repetitions, and seeking behaviors. Am J Occup Ther. 2015;69(3):1-9.

10. Schauder KB, Muller CL, Veenstra-Vanderweele J, Cascio CJ. Genetic variation in serotonin transporter modulates tactile hyperresponsiveness in ASD. Res Autism Spectr Disord. 2015;10:93-100.

11. Cascio CJ, Gu C, Schauder KB, Key AP, Yoder P. Somatosensory event-related potentials and association with tactile behavioral responsiveness patterns in children with ASD. Brain Topogr. 2015;28:895-903.

12. Pryweller JR, Schauder KB, Anderson AW, Heacock JL, Newsom CR, Loring WA, Cascio CJ. White matter correlates of sensory processing in autism spectrum disorders. Neuroimage: Clinical. 2014;6:379-87.

13. Ausderau KK, Furlong M, Sideris J, Bulluck J, Little LM, Watson LR, Boyd BA Belger A, Dickie VA, Baranek GT. Sensory subtypes in children with autism spectrum disorder: latent profile transition analysis using a national survey of sensory features. J Child Psychol Psychiatry. 2014;55:935-44.

14. Hertenstein MJ, Verkamp JM, Kerestes AM, Holmes RM. The communicative function of touch in humans, nonhuman primates, and rats: a review and synthesis of the empirical research. Genet Soc Gen Psychol Monogr. 2006; 94:132-5.

15. Duhn L. The importance of touch in the development of attachment. Advances in Neonatal Care. 2010;10(6):294-300.

16. Main M, Stadtman J. Infant response to rejection of physical contact by the mother: aggression, avoidance, and conflict. J American Acad Child Psychiatry. 1981;20:292-307.

17. Gallace A, Spence C. The science of interpersonal touch: an overview. Neurosci Biobehav Rev. 2010;34(2):246-59. 
18. Wolff J, Gu H, Gerig G, Elison JT, Styner M, Gouttard S, Botteron KN, Dager SR, Dawson G, et al. Differences in white matter fiber tract development present form 6 to 24 months in infants with autism. Am J of Psychiatry. 2012;169(6):589-600

19. Mikkelsen M, Wodka EL, Mostofsky SH, Puts NA. Autism spectrum disorder in the scope of tactile processing. Dev Cog Neurosci. 2016; [Epub ahead of print] doi: 10.1016/den.2016.12.005

20. Puts NA, Wodka EL, Tommerdahl M, Mostofsky SH, Edden RA. Impaired tactile processing in children with autism spectrum disorder. J Neurophysiol. 2014;112(6):1600-1.

21. Tavassoli T, Bellesheim K, Tommerdahl M, Holden JM, Kolevzon A, Buxbaum JD. Altered tactile processing in children with autism spectrum disorder. Autism Res. 2016:9(6):616-20.

22. Tommerdahl M, Tannan V, Cascio CJ, Baranek GT, Whitsel BL. Vibrotactile adaptation fails to enhance spatial localization in adults with autism. Brain Res. 2007;1154:116-23.

23. Puts NA, Wodka EL, Harris AD, Crocetti D, Tommerdahl M, Mostofsky SH, Edden RA. Reduced GABA and altered somatosensory function in children with autism spectrum disorder. Autism Res. 2016; Sep 9. doi: 10.1002/aur. 1691. [Epub ahead of print]

24. Uzunova G, Pallanti S, Hollander E. Excitatory/inhibitory imbalance in autism spectrum disorders: implications for interventions and therapeutics. World J Biol Psychiatry. 2016;17(3):174-86.

25. Foss-Feig JH, Adkinson BD, Ji JL, Yang G, Srihan VH, McPartland JC, Krystal $J H$, Murray JD, Anticevic A. Searching for cross-diagnostic convergence: neural mechanisms governing excitation and inhibition balance in schizophrenia and autism spectrum disorders. Biol Psychiatry. 2017;81(10): 848-61. http://doi.org/10.1016/j.biopsych.2017.03.005

26. DeLorey TM, Sahbaie P, Hashemi E, Li WW, Salehi A, Clark DJ. Somatosensory and sensorimotor consequences associated with the heterozygous disruption of the autism candidate gene, GABRB3. Behav Brain Res. 2011;216(1):36-45.

27. Tavassoli T, Auyeung B, Murphy LC, Baron-Cohen S, Chakrabarti B. Variation in the autism candidate gene GABRB3 modulates tactile sensitivity in typically-developing children. Mol Autism. 2012;3(1):6.

28. Cohen LG, Bandinelli S, Sato S, Kufta C, Hallet M. Attenuation in detection of somatosensory stimuli by transcranial magnetic stimulation. Electroencephalogr Clin Neurophysiol. 1991;81(5):336-76.

29. Knecht S, Ellger T, Breitenstein C, Bernd Ringelstein E, Henningsen $H$. Changing cortical excitability with low-frequency transcranial magnetic stimulation can induce sustained disruption of tactile perception. Biol Psychiatry. 2003;53(2):175-9.

30. Lundblad LC, Olausson HW, Hermansson AK, Wasling HB. Cortical processing of tactile direction discrimination based on spatiotemporal cues in man. Neurosci Lett. 2011:501(1):45-9.

31. McGlone F, Wessberg J, Olausson H. Discriminative and affective touch: Sensing and feeling. Neuron. 2014;82(4):737-55.

32. Olausson H, Lamarre $Y$, Backlund H, Morin C, Wallin BG, Starck G, Ekholm S, Strigo I, Worsley $\mathrm{K}$, et al. Unmyelinated tactile afferents signal touch and project to insular cortex. Nat Neurosci. 2002;5(9):900-4.

33. Olausson HW, Cole J, Vallbo A, McGlone F, Elam M, Kramer H, Rylander K, Wessberg J, Bushnell MC. Unmyelinated tactile afferents have opposite effects on insular and somatosensory cortical processing. Neurosci Lett. 2008;436(2):128-32.

34. Morrison I, Bjornsdotter M, Olausson H. Vicarious responses to social touch in posterior insular cortex are tuned to pleasant caressing speeds. J Neurosci. 2011;31(26):9954-562.

35. Mesulam MM, Mufson EJ. Insula of the old world monkey III: efferent cortical output and comments on function. J Comp Neurol. 1982;212:38-52.

36. Simmons WK, Avery JA, Barcalow JC, Bodurka J, Drevets WC, Bellgowan O. Keeping the body in mind: insula functional organization and functional connectivity integrate interoceptive, exteroceptive, and emotional awareness. Hum Brain Mapp. 2013;34(11):2944-58.

37. Craig AD. How do you feel? Interoception: the sense of the physiological condition of the body. Nat Rev Neurosci. 2002;3:655-66.

38. Farb NA, Degal ZV, Anderson AK. Attentional modulation of primary interoceptive and exteroceptive cortices. Cereb Cortex. 2013;23:114-26.

39. Menon V, Uddin LQ. Saliency switching, attention, and control: a network model of insula function. Brain Struct Funct. 2010;214(5-6):655-67.

40. Uddin LQ, Menon V. The anterior insula in autism: under-connected and under-examined. Neurosci Biobehav Rev. 2009;33(8):1998-203.
41. Odriozola P, Uddin LQ, Lunch CJ, Kochalka J, Chen T, Menon V. Insula response and connectivity during social and non-social attention in children with autism. Soc Cogn Affect Neurosci. 2016;11(3):433-44.

42. Gliga $\mathrm{T}$, Jones EJ, Bedford R, Charman $\mathrm{T}$, Johnson MH. From early markers to neurodevelopmental mechanisms of autism. Dev Rev. 2014;34(3):189-207.

43. Cascio CJ, Woynaroski T, Baranek GT, Wallace MT. Toward an interdisciplinary approach to understanding sensory function in autism spectrum disorder. Autism Res. 2016;9(9):920-5.

44. Cascio CJ, Moana-Filho EJ, Guest S, Nebel MB, Weisner J, Baranek GT, Essick GK. Perceptual and neural response to affective tactile texture stimulation in adults with autism spectrum disorders. Autism Res. 2012;5(4):231-44.

45. Fiene $\mathrm{L}$, Browlow C. Investigating interoception and body awareness in adults with and without autism spectrum disorder. Autism Res. 2015;8(6):709-16.

46. Schauder KB, Mash LE, Bryant LK, Cascio CJ. Interoceptive ability and body awareness in autism spectrum disorder. J Exp Child Psychol. 2015:131:193-200.

47. Mash LE, Schauder KB, Cochran C, Park S, Cascio CJ. The development of interoceptive cognition in autism spectrum disorder and typical development. J Cog Ed Psychol. 2017;16:23-37.

48. Shukla DK, Keehn B, Muller RA. Tract-specific analyses of diffusion tensor imaging show widespread white matter compromise in autism spectrum disorder. J Child Psychol Psychiatry. 2011:52(3):286-95.

49. Vogan VM, Morgan BR, Leung RC, Anagnostou E, Doyle-Thomas K, Taylor MJ. Widespread white matter differences in children and adolescents with autism spectrum disorder. J Autism Dev Disord. 2016;46(6):2138-47.

50. Lord C, Rutter M, DiLavore P, Risi S. The Autism Diagnostic Observation Schedule (ADOS). Western Psychological Services; 1999.

51. Lord C, Rutter M, Le Couteur A. Autism Diagnostic Interview-Revised: a revised version of a diagnostic interview for caregivers of individuals with possible pervasive developmental disorders. J Autism Dev Disord. 1994;24:659-85.

52. American Psychiatric Association. Diagnostic and statistical manual of mental disorders (4th ed.). 4th, text rev. ed. Washington, DC; 2000.

53. Rutter M, Bailey AJ, Lord C. The social communication questionnaire. Los Angeles: Western Psychological Services; 2003.

54. Achenbach TM, Rescorla LA. Manual for the ASEBA school age forms and profiles. Burlington: VT; 2001.

55. Kaufman AS, Kaufman NL. KBIT2: Kaufman Brief Intelligence Test-II. 2nd ed. Circle Pines: AGS Publishing; 2004.

56. Wechsler D, Kaplan E, Fein D, Kramer J, Morris R, Delis D, Maelender A Wechsler intelligence scale for children: fourth edition (WISC-IV). San Antonio: Pearson; 2003.

57. Mullen EM. Mullen Scales of Early Learning. AGSth ed. American Guidance Service: Circle Pines; 1995.

58. Lauzon CB, Asman AJ, Esparza ML, Burns SS, Fan Q, Gao Y, et al. Simultaneous analysis and quality assurance for diffusion tensor imaging. PLoS One. 2013;8(4):e61737.

59. Jenkinson M, Beckmann CF, Behrens TE, Woolrich MW. Smith SM. FSL Neuroimage. 2012;62:782-90.

60. Smith SM, Jenkinson M, Woolrich MW, Beckmann CF, Behrens TE, JohansenBerg H, Bannister PR, De Luca M, Dobnjak I, et al. Advances in functional and structural MR image analyses and implementation in FSL. Neuroimage. 2004;23 Suppl 1:S208-19.

61. Behrens TE, Beg HJ, Jbabdi S, Rushworth MF, WOolrich MW. Probabilistic diffusion tractography with multiple fibre orientations: What can we gain? Neuroimage. 2007:34:144-55.

62. Fonov V, Evans A, McKinistry R, Almi C, Collins D. Unbiased nonlinear average age-appropriate brain templates from birth to adulthood. Neurolmage. 2009;47:S102.

63. Fonov V, Evans AC, Botteron K, Almli CR, McKinstry RC, Collins DL. Brain Development Cooperative Group. Unbiased average age-appropriate atlases for pediatric studies Neurolmage. 2011;54:313-27.

64. Johansen-Berg H, Behrens TEJ, Sillery EL, Ciccarelli O, Wheeler-Kingshott CAM, Thompson AJ, Smith SM, Matthews PM. Functional Anatomical validation and individual variation of diffusion tractography-based segmentation of the human thalamus. Cereb Cortex. 2005;15(1):31-9.

65. Shattuck DW, Mizra M, Adisetiyo V, Hojatkashani C, Salamon G, Narr KL, Poldrack RA, Bilder RM, Toga AW. Construction of a 3D probabilistic atlas of human cortical structures. Neurolmage. 2008;39:1064-80.

66. Klein A, Andersson J, Ardekani BA, Ashburner J, Avants B, Chiang MC, Christensen MC, Christensen GE, et al. Evaluation of 14 nonlinear deformation algorithms applied to human brain MRI registration. Neurolmage. 2009;46:786-802. 
67. Tustison NJ, Cook PA, Klein A, Song G, Das SR, Duda JT, Kandel BM, van Strien N, et al. Large-scale evaluation of ANTs and FreeSurfer cortical thickness measurements. Neurolmage. 2014;99:166-79.

68. Cullen KR, Klimes-Dougan B, Muetzel R, Mueller BA, Camchong J, Houi A, Kurma S, Lim KO. Altered white matter microstructure in adolescents with major depression: a preliminary study. J Am Acad Child Adolesc Psychiatry. 2010;49:173-83. e1.

69. Lim JC, Phal PM, Desmond PM, Nichols AD, Kokkinos C, Danesh-Meyer HV, Kaye AH, Moffat BA. Probabilistic MRI tractography of the optic radiation using constrained spherical deconvolution: a feasibility study. PLoS One. 2015;10:e0118948.

70. Dudoit S, Shaffer JP, Boldrick JC. Multiple hypothesis testing in microarray experiments. Stat Sci. 2003;18:71-103.

71. Cascio C, McGlone F, Folger S, Tannan V, Baranek G, Pelphrey KA, Essick G. Tactile perception in adults with autism: a multidimensional psychophysical study. J Autism Dev Disord. 2008;38(1):127-37.

72. Haigh SM, Minshew N, Heeger DJ, Dinstein I, Behrmann M. Overresponsiveness and greater variability in roughness perception in autism. Autism Res. 2016;9:393-402.

73. Riquelme I, Hatem SM, Montoya P. Abnormal pressure pain, touch sensitivity, proprioception, and manual dexterity in children with autism spectrum disorders. Neural Plast. 2016; e1723401.

74. Liljencrantz J, Olausson $\mathrm{H}$. Tactile C fibers and their contributions to pleasant sensations and to tactile allodynia. Front Behav Neurosci. 2014;8:37.

75. Kaiser MD, Yan DY, Voos AC, Bennet RH, Gordon I, Pretzsch C, Beam D,

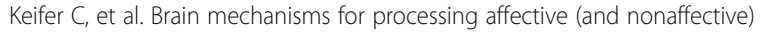
touch are atypical in autism. Cereb Cortex. 2016;26:2705-14.

76. Scheele D, Striepens N, Gunturkun O, Deutschlander S, Maier W, Kendrick KM, Hurlemann R. An oxytocin-induced facilitation of neural and emotional responses to social touch correlates inversely with autism traits. Neuropsychopharmacol. 2014;39:2078-85.

77. Voos AC, Pelphrey KA, Kaiser MD. Autistic traits are associated with diminished neural response to affective touch. Soc Cogn Affect Neurosci. 2013:8:378-86

78. Engel-Yeger B, Dunn W. The relationship between sensory processing difficulties and anxiety level of healthy adults. Br J Occup Ther. 2011;74(5): 210-16.

79. Higashida N. The Reason I Jump. New York: Random House; 2013

80. Pfeiffer B, Kinnealey M, Reed C, Herzberg G. Sensory modulation and affective disorders in children and adolescents with Asperger's disorder. Am J Occup Ther. 2005;59:335-45.

81. Dunn W. The sensations of everyday life: empirical, theoretical, and pragmatic considerations. Am J Occup Ther. 2011;55:608-20.

82. Liss M, Saulnier C, Fein D, Kinsbourne M. Sensory and attention abnormalities in autistic spectrum disorders. Autism Int J Res Pract. 2006;10:155-72.

83. Boyd BA, Baranek GT, Sideris J, Poe MD, Watson LR, Patten E, Miller H. Sensory features and repetitive behaviors in children with autism and developmental delays. Autism Res. 2010;3:78-87.

84. Cascio CJ, Foss-Feig JH, Heacock J, Schauder KB, Loring WA, Rogers BP, Pryweller JR, Newsom CR, et al. Affective neural response to restricted interests in autism spectrum disorders. J Child Psychol Psychiatry. 2014;55: $162-71$.

85. Dichter GS, Felder JN, Green SR, Rittenberg AM, Sasson NJ, Bodfish JW Reward circuitry function in autism spectrum disorders. Soc Cog Affect Neurosci. 2012;7:160-72.

86. Mayer EA. Gut feelings: the emerging biology of gut-brain communication. Nat Rev Neurosci. 2011;12(8):453-66.

87. Segerdahl AR, Mezue M, Okell TW, Farra JT, Tracey I. The dorsal posterior insula subserves a fundamental role in human pain. Nat Neurosci. 2015; 18(12):1861.

88. Failla MD, Moana-Filho EJ, Essick GK, Baranek GT, Rogers BP, Cascio CJ. Initially intact neural responses to pain in autism are diminished during sustained pain. Autism. (in press); DOl: 10.1177/1362361317696043.

89. Bonenberger M, Plener PL, Groschaitz RC, Gron G, Abler B. Differential neural processing of unpleasant haptic sensations in somatic and affective partitions of the insula in non-suicidal self-injury (NSSI). Psychiatry Res. 2015; 234(3):298-304.

90. Stoesz MR, Zhang M, Weisser VD, Prather SC, Mao H, Sathian K. Neural networks active during tactile form perception: common and differential activity during macrospatial and microspatial tasks. Int J Psychophysiol. 2003;50:41-9.
91. Trotter PD, McGlone F, McKie S, McFarquhar M, Ellitt R, Walker SC, Deakin JF. Effects of acute tryptophan depletion on central processing of CT-targeted and discriminatory touch in humans. Eur J Neurosci. 2016;44(4):2072-83.

92. Wang $W$, Zhang $M$, Wang $Y$, Jin C, Yan B, Ma S. 5-HT modulation of pain in SI and SII revealed by fMRI. Zhong Nan Da Xue Xue Bao Yi Xue Ban. 2010; 35(3):185-93.

93. Al B, Dietrich MS, Blackford JU, Charboneau EJ, Lillevig JG, Cannistraci CJ, Woodward ND, Cao A, et al. Human ecstasy use is associated with increased cortical excitability: an fMRI study. Neuropsychopharmacology. 2011;36(6):1127-41.

94. Cook EH, Leventhal BL. The serotonin system in autism. Curr Opin Pediatr. 1996;8(4):348-54.

95. Veenstra-VanderWeele J, Muller $\mathrm{CL}$, Iwamoto $\mathrm{H}$, Sauer JE, Owens WA, Shah CR, Coehn J, Mannangatti P, Jessen T, et al. Autism gene variant causes hyperserotonemia, serotonin receptor hypersensitivity, social impairment and repetitive behavior. Proc Natl Acad Sci U S A. 2012;109(14):5469-74.

96. Ciranna L. Serotonin as a modulator of glutamate and GABA-mediated neurotransmission: implications in physiological functions and in pathology. Curr Neuropharmacol. 2006;4(2):101-14.

97. Kida T, Shinohara K. Gentle touch activates the prefrontal cortex in infancy: an NIRS study. Neurosci Lett. 2013;541:63-6.

\section{Submit your next manuscript to BioMed Central and we will help you at every step:}

- We accept pre-submission inquiries

- Our selector tool helps you to find the most relevant journal

- We provide round the clock customer support

- Convenient online submission

- Thorough peer review

- Inclusion in PubMed and all major indexing services

- Maximum visibility for your research

Submit your manuscript at www.biomedcentral.com/submit
Biomed Central 